\title{
Isquemia miocárdica con coronarias de aspecto angiográfico normal. Enfoque diagnóstico.
}

Miǵuel Lópes-Hidalgo ${ }^{1,2}$ y Antonio Eblen-Zajjur 1, 2,3

1 Área de Postgrado, Doctorado en Ciencias Médicas de la Universidad de Carabobo, Valencia, Venezuela.

${ }^{2}$ Centro de Biofísica y Neurociencia, Facultad de Ciencias de la Salud, Universidad de Carabobo, Valencia, Venezuela.

${ }^{3}$ Universidad Diegoo Portales, Dirección de Postǵrado, Santiaǵo, Chile.

Palabras clave: síndrome X cardíaco; disfunción microvascular coronaria; angina microvascular; espasmo coronario microvascular; enfermedad arterial coronaria; dolor torácico.

Resumen. Los pacientes con angina de pecho, evidencia de isquemia miocárdica y angiografía con coronarias no obstructivas, implican un verdadero reto diaǵnóstico. Estudios recientes han demostrado que, en esta entidad clínica, el pronóstico no es tan benigno como se pensaba. El síndrome X cardíaco, actualmente más conocido como isquemia miocárdica y arterias coronarias no obstructivas (INOCA: Ischemia and No Obstructive Coronary Arteries), se ha enfocado predominantemente en la disfunción microvascular, sin embargoo, los otros mecanismos causales como la enfermedad aterosclerótica oculta, el espasmo de coronarias epicárdicas y el síndrome del corazón sensible, siempre deben ser considerados. Esta revisión basada en la evidencia de los últimos 20 años, plantea una visión práctica del síndrome INOCA. Se aborda la inquietud y esfuerzos de los diferentes investigadores que estudian este síndrome, con la finalidad de lograr un protocolo diagnóstico adecuado, para así orientar mejor la terapéutica según el mecanismo fisiopatológico predominante.

Autor de Correspondencia: Miǵuel López Hidalgoo. Universidad de Carabobo, FCS, Área de postǵrado, Proǵrama de Doctorado en Ciencias Médicas, Valencia, Carabobo, Venezuela. Correo electrónico: lopezh900@hotmail.com 


\title{
Myocardial ischemia and coronary arteries with normal angiographic appearance. Diagnostic approach.
}

\author{
Invest Clin 2020; 61 (4): 376-392
}

Key words: cardiae syndrome $\mathrm{X}$; coronary microvascular dysfunction; microvaseular angina; coronary microvascular spasm; coronary artery disease; chest pain.

\begin{abstract}
Patients with angina, evidence of myocardial ischemia and angiography with no obstructed coronary arteries represent a real diagnostic challenge to the clinical cardiologist. Recent evidence has shown that this entity is not as benign as it was thought. Cardiac syndrome X, currently known as myocardial Ischemia with No Obstructed Coronary Arteries (INOCA), has focused predominantly in microvascular dysfunction. However, the other causal mechanisms, such as concealed atherosclerotic coronary artery disease, epicardial coronary spasm and the sensitive heart syndrome, should always be considered. This evidence-based review of the last 20 years of publications raises a practical vision of INOCA syndrome. It approaches the concerns and efforts of investigators who study this syndrome to achieve a proper diagnostic protocol, in order to guide a more appropriate treatment approach according to the predominant pathophysiologic mechanism.
\end{abstract}

Recibido: 26-07-2020 Aceptado: 24-10-2020

\section{INTRODUCCIÓN}

Los pacientes con angina de pecho y angiografía coronaria sin lesiones obstructivas significativas, constituyen un verdadero reto para el clínico, ya que no pocas veces se considera que el dolor es de origen no cardíaco y por lo tanto el abordaje diagnóstico y terapéutico conduce a errores y fracaso. La prevalencia de esta condición se reportó inicialmente como 18,8\% según el estudio CASS (Coronary Artery Surgery Study). El registro SCAI (Society for Cardiac Angiography and Intervention) de 1993 encontró que 20 a $27 \%$ de los pacientes tenía angiografía coronaria de aspecto normal y un 35\% presentaba menos de $50 \%$ de estenosis angiográfica, esta última condición catalogada como "enfermedad arterial coronaria no obstructiva" (1). Más recientemente, en un estudio de 400.000 pacientes, esta prevalencia ha sido reportada como $59 \%$, lo cual resalta su importancia epidemiológica (2). La prevalencia del síndrome X cardíaco (SXC) en la mujer es 2 a 3 veces mayor que en el hombre $(3,4)$. Hasta no hace poco se consideraba que el pronóstico del SXC era benigno y se recomendaban medidas de apoyo, asegurando al paciente, que su condición era de bajo riesgo. Nueva evidencia apunta a que la naturaleza de este síndrome no es tan benigna como se pensaba (5). A pesar de esto, actualmente no existen guías basadas en la evidencia para el manejo de estos pacientes $(3,6)$.

Existe un cúmulo de evidencias de más de 45 años, que muestran la relevancia clínica del SXC, descrito por primera vez por Arbogast y col. en 1973 (7-9). En los últimos

Vol. 61(4): 376 - 392, 2020 
años la atención sobre el SXC se ha enfocado en la investigación de la disfunción de la microcirculación coronaria, con menor énfasis en los otros mecanismos fisiopatológicos. Desde hace más de 20 años el grupo de Bairey Merz, viene trabajando para avanzar en la investigación de esta entidad, con enfoque basado en la evidencia, para unificar y estandarizar criterios diagnósticos. Los términos más utilizados actualmente para este síndrome, de acuerdo con estos autores son: Isquemia miocárdica y Arterias Coronarias No Obstructivas o INOCA y Angina microvascular $(6,10)$. El SXC o INOCA se define como aquellos pacientes que presentan: 1) Angina de pecho inducida por esfuerzo, 2) Alteraciones del segmento ST durante la angina 3) Anǵiografía con coronarias epicárdicas de aspecto normal, 4) Test de provocación de vasoconstricción epicárdica negativo 5) Ausencia de enfermedad cardíaca o sistémica con disfunción microvascular secundaria, como la miocardiopatía hipertrófica o la diabetes (11).

Esta revisión plantea una visión clínica y práctica del paciente con dolor de pecho, con evidencia de isquemia miocárdica y coronarias de aspecto angiográfico normal, haciendo énfasis en los mecanismos fisiopatológicos involucrados y en el diaǵnóstico etiológico adecuado, para un mejor abordaje terapéutico.

\section{MÉTODO}

Es una revisión sistemática, descriptiva, con énfasis en el diagnnóstico del síndrome de angina de pecho estable con coronarias de aspecto normal. Se seleccionó la literatura utilizada con amplitud en tiempo, para los datos históricos, y para los datos diaǵnósticos solo se consultaron los últimos 20 años, debido al creciente interés en la investigación de esta entidad en este lapso. Se utilizó la metodología de cadena de búsqueda booleana, con sus conectores apropiados, con buscadores electrónicos generales iniciales como Google, para obtener datos más amplios con términos generales y luego buscadores especializados en Medicina, para resultados más específicos como: Pubmed, Cochrane, Google Scholar, Medscape Cardiology y Scielo, en los idiomas inglés y español. Se utilizaron los siguientes términos MESH con los buscadores especializados: síndrome $\mathrm{X}$ cardíaco, angina microvascular (AMV), disfunción microvascular (DMV), anǵina vasoespástica (AVE) y angina estable, concatenados con los términos diagnóstico o enfoque diaǵnóstico; INOCA (ischemia and no obstructive coronary arteries) no aparece como término MESH, por lo que en este caso se utilizó un buscador más genérico, como Google. En la búsqueda se encontraron 3.600 publicaciones para SXC, 870 para DMV, 1.350 para AMV, 2890 para angina vasoespástica y 2150 para INOCA. Se descartaron 9.960, las que coincidían entre los mismos términos y las duplicadas en los diferentes buscadores, quedando 900 en total. De estas, se seleccionaron todas las revisiones y los estudios específicos sobre diagnóstico, relativos al tema, quedando 186 referencias. Para la elaboración del manuscrito se seleccionaron las 48 más pertinentes, respetando los límites de las normativas de publicación.

\section{ABORDAJE DIAGNÓSTICO INICIAL}

En presencia de un paciente con angina de pecho, con evidencia objetiva de isquemia miocárdica por estudios no invasivos (prueba de esfuerzo, ecocardiograma stress) y con angiografía coronaria sin lesiones, la primera pregunta que nos debemos hacer es la siguiente: ¿Es verdaderamente normal la angiografía coronaria?

Ante esto se plantea: hacer una nueva revisión bien detallada de la angiografía coronaria que pudiera develar el diaǵnóstico preciso, cuando se buscan intencionalmente lesiones ocultas o difíciles de precisar. Si no se logra detectar lesiones y existe un alto índice de sospecha de isquemia miocárdica, Schuartz y col. (14) sugieren la utilización del siguiente protocolo de evaluación (Fig. 1). 


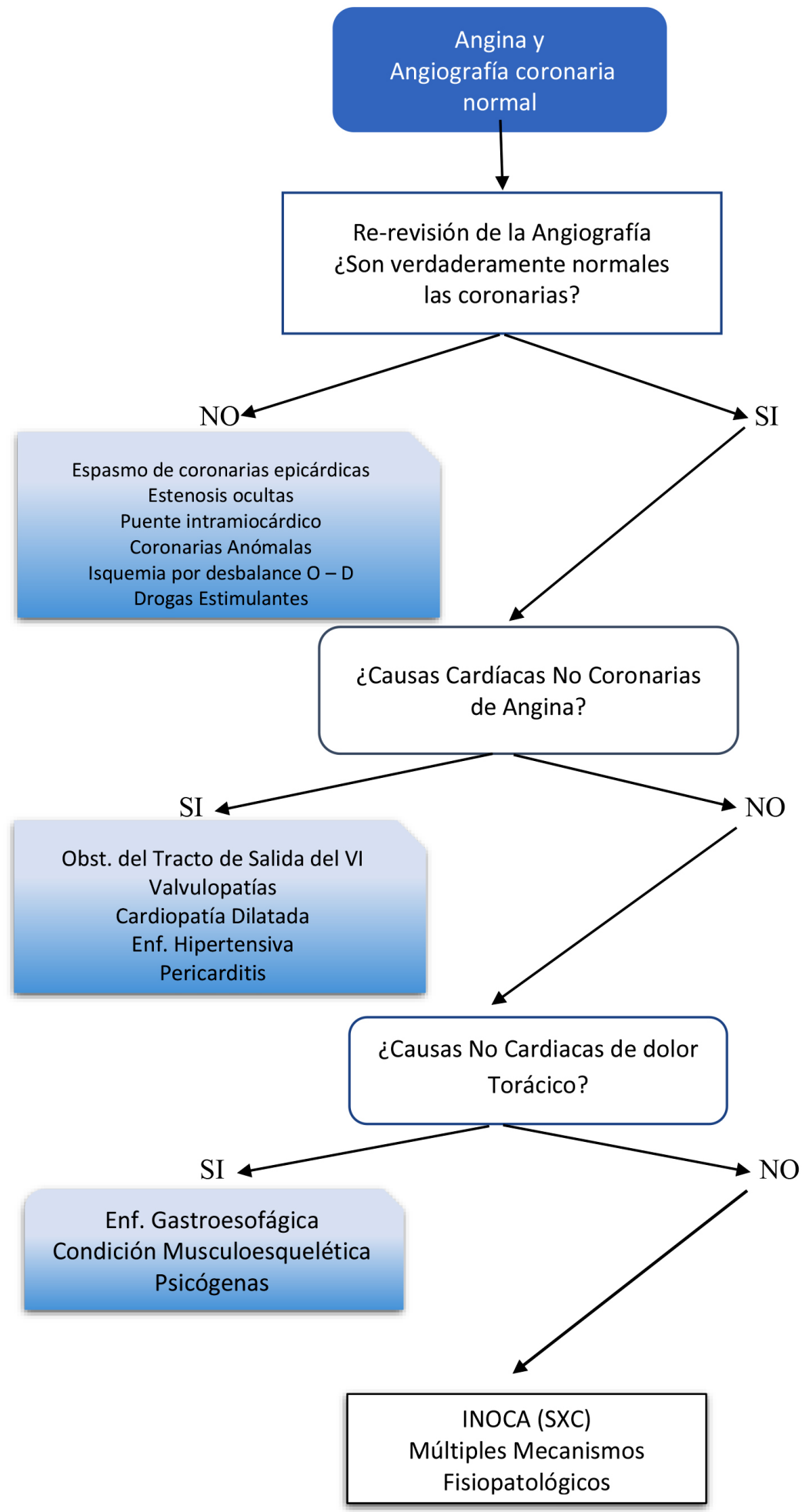

Fig. 1. Algoritmo Diaǵnóstico en el Síndrome INOCA (SXC).

Modificado de Schuartz y col., 2001 (14). 
Causas originadas en las arterias coronarias epicárdicas

\section{Estenosis coronarias ocultas o inad- vertidas}

La angiografía coronaria tiene sus limitaciones para la detección de ciertos tipos de lesiones obstructivas, principalmente porque lo que visualizamos con el contraste, es la luz del vaso sin detalles de la pared vascular. La reevaluación visual de la angiografía debe centrarse en buscar lesiones en sitios donde pueden ser difíciles de visualizar o aparezcan escondidas, como las lesiones aorto-ostiales y en ostia de bifurcaciones donde las angulaciones de las proyecciones, muchas veces no logran captar la magnitud de la estenosis, particularmente en lesiones excéntricas, pudiendo aparecer en ocasiones lesiones muy severas y en otras lesiones no significativas con falsos resultados; esto también puede ocurrir en lesiones localizadas en segmentos con curvaturas y angulaciones naturales de las arterias coronarias y cuando existen proyecciones donde hay ramos sobrepuestos en el sitio de una lesión y que a pesar de haber hecho varias proyecciones en diferentes ángulos, no se logra separar los ramos para poder visualizar dicha lesión. Es allí cuando es necesario aplicar otras técnicas de imágenes que pudieran detectar la presencia o no de lesión coronaria signnificativa. Estas técnicas son el ultrasonido intravascular (IVUS, por sus siǵlas en inglés: Intra-Vascular UltraSound), la tomografía de coherencia óptica que permite ver cortes tomográficos axiales muy finos y así visualizar la distribución de la placa ateromatosa en la pared del vaso, para mejor evaluar y medir el compromiso de la luz, complementando así la información anatómica de la anǵiografía y aumentando el poder de detección de las lesiones ocultas, tales como las ya descritas (14). La evaluación funcional de las lesiones, que mide la reserva de flujo fraccional coronario (FFR: Fractional Flow Reserve), que se obtiene con la relación entre la presión intracoronaria distal a la lesión y la presión de la aorta proximal, en condición basal y posterior a hiperemia con adenosina, nos permite detectar con precisión las lesiones angiográficas hemodinámicamente signnificativas (es decir las que producen isquemia miocárdica), esto es cuando el FFR es $<0,80$ y se utiliza especialmente en aquellas estenosis angiográficas intermedias, definidas como estenosis entre $50 \%$ a $70 \%$, esta herramienta también puede ser útil en aquellas estenosis que están ocultas o escondidas como previamente descrito. Recientemente se ha desarrollado el índice de relación instantánea libre de onda (iFR: Instantaneous Free Wave Ratio), que realiza la misma evaluación sin la necesidad de inducir hiperemia con adenosina, lo cual es más práctico, rápido y con alta correlación con el FFR. Las lesiones del tronco principal de la arteria coronaria izquierda (TPCI), son difíciles de evaluar solo con angiografía, por lo que el IVUS y el FFR son esenciales. Publicaciones sobre evaluaciones dedicadas al TPCI, han demostrado que la sensibilidad para detectar lesiones significativas en el tercio medio y distal es mayor con el FFR que con el IVUS (14-16).

La angio-tomografía computarizada cardiaca, es también una herramienta diaǵnóstica valiosa no invasiva, ya que proporciona una visión topográfica tridimensional, muy útil en todos los tipos de lesiones mencionados, particularmente, en lesiones ocultas por ramos en la angiografía y permite visualizar la distribución de la placa de ateroma en la pared vascular, y proporciona algunos detalles en cuanto al remodelado vascular, tales como las características y distribución del ateroma, si la placa es blanda o dura (fibrótica) y el grado de calcificación. Adicionalmente, se ha añadido a la angioTAC cardiaca, la evaluación de la reserva del flujo fraccional coronario (FFR-CT: Fractional Flow Reserve with Computed Tomography), que da una evaluación funcional derivada de algoritmos matemáticos complejos, basada en la dinámica de fluido del contraste y con el mismo principio del FFR invasivo, mostrando un mapa del árbol coronario 
anǵiográfico a colores, generalmente verde el segmento coronario normal y en rojo el seǵmento a partir del cual se encuentra la lesión hemodinámicamente significativa, facilitando así su interpretación. Este método fue aprobado por la administración de drogas y alimentos de Norteamérica (FDA: Food and Drug Administration) en 2013, para la evaluación de pacientes con sospecha de enfermedad arterial coronaria estable, que presenten estenosis intermedias (50 a 69\%) en la Angiotac cardíaca y cuando el FFR-CT es normal $>0,80$. La gran ventaja de esto, es que permite diferenciar aquellos pacientes que no van a requerir más evaluaciones ni intervenciones. Los autores que recientemente han aplicado FFR-CT en estudios de series de pacientes, llegaron a la conclusión, que se requieren otros estudios aleatorizados con seguimiento a mayor plazo, para evaluar la capacidad predictiva, y definir la estrategia óptima de manejo de pacientes estratificados con AngioCT y FFR-CT $(6,17)$, por lo que habría que esperar hasta obtener datos suficientes, para considerar el FFR-CT como un estudio que pueda sustituir la FFR por cateterismo. Es de hacer notar que hasta el momento no se ha realizado ningún estudio en el síndrome INOCA, utilizando el FFR-CT, lo cual pudiera ser una vez validada, una opción de gran utilidad.

\section{Espasmo coronario}

La angina vasoespástica (AVE) o de Prinzmetal, es de carácter transitorio, cíclico, con comportamiento circadiano; se produce en reposo, con elevación transitoria del segmento ST en el electrocardiograma (ECG) y se puede provocar con la hiperventilación. Generalmente no es inducido por el ejercicio, por lo que no se reproduce en las pruebas de esfuerzo, lo cual puede producir confusión. Se especula que por esta razón se subvalora y no se registra. Su diaǵnóstico definitivo se realiza con las pruebas de provocación de vasoconstricción coronaria (PPVCG) con acetilcolina y/o ergonovina durante la angiografía coronaria. La acetilcolina actúa como neurotransmisor parasimpático en los receptores muscarínicos endoteliales, activando la liberación de óxido nítrico $(\mathrm{ON})$ y produciendo vasodilatación, pero si hay disfunción o denudación del endotelio vaseular, con falla o ausencia en la producción de ON, se produce estimulación directa de los receptores muscarínicos tipo 3 del músculo liso vascular con respuesta vasoconstrictora. La ergoonovina actúa con un mecanismo similar y en condiciones normales, existe un equilibrio entre las prostaglandinas vasodilatadoras secretadas por el endotelio sano y la erǵonovina, evitando la vasoconstricción; pero cuando existe disfunción endotelial, con falla de producción de la prostaglandina, la ergonovina actúa directamente sin oposición, en los receptores serotoninérgiicos del músculo liso vascular, produciendo vasoconstricción $(18,19)$.

Los criterios diaǵnósticos para la AVE son: las características clínicas ya mencionadas y la presencia de vasoespasmo coronario, con constricción del vaso mayor de $90 \%$, con angina de pecho y desnivel del segmento ST en el ECG después de la inyección intracoronaria (IC) de 20 a $100 \mu g$ de acetilcolina en 20 segundos o con el uso de 20 a $60 \mu \mathrm{g}$ de ergonovina IC en 20 a 30 segundos. El uso endovenoso de la ergónovina, no se recomienda por que puede producir espasmo coronario prolongado y multiarterial con riesgo de muerte (19). Varios investigadores entre ellos los del COVADIS, grupo internacional de estudios de las alteraciones vasomotoras coronarias, que preconizan el uso de las PPVCC, recientemente, publicaron una guía que sirve de estándar para su uso, donde especifican las indicaciones según el grado de evidencias y su grado de seguridad (20) (Tabla I). La indicación principal de las PPVCG es la alta sospecha de AVE según las características ya descritas. Esta guía fue utilizada en el estudio prospectivo más completo hasta ahora, sobre el síndrome INOCA, el estudio CorMica, donde ratifican la utilidad y seguridad de las PPVCG. La prevalencia de AVE en este estudio fue de $17 \%(21,26)$. En

Vol. 61(4): 376 - 392, 2020 
TABLA I

INDICACIÓN PARA PRUEBA DE PROVOCACIÓN DE VASOCONSTRICCIÓN CORONARIA.

\begin{tabular}{ll}
\hline Tipo de Indicación & \multicolumn{1}{c}{ Criterio Clínico } \\
\hline & Sospecha de AVE sin episodio documentado, en presencia de: \\
& - Angina en reposo con buena respuesta a nitratos \\
& - Marcada variación circadiana de inicio de síntomas \\
& - Buena tolerancia al ejercicio \\
& - Angina en reposo con arterias coronarias de aspecto normal \\
& - Sin respuesta a la terapia empírica de INOCA \\
& - SCA con arterias coronarias sin lesiones obstructivas \\
Clase I (Fuerte) & - Paro cardíaco resucitado, sin causa definida \\
& - Síncope precedido de dolor torácico sin causa definida \\
& - Angina recurrente seguida de ICP exitoso \\
& - Pacientes con diagnóstico no invasivo, sin respuesta al tratamiento \\
& - Episodio documentado de AVE espontanea, para determinar el sitio \\
Clase IIa (Aceptable) & afectado, la severidad y su mecanismo \\
& - Pacientes con diagnóstico no invasivo, con respuesta al tratamiento \\
Clase IIb (Controversial) & - Sospecha de AVE con disfunción ventricular izquierda moderada \\
& - SCA con lesiones obstructivas \\
Clase III (Contraindicación) & - EAC multiarterial \\
& - Disfunción ventricular izquierda severa \\
& - Paciente sin síntomas sugestivos de AVE \\
\hline
\end{tabular}

AVE: Angina Vasoespastica; INOCA: Isquemia y arterias coronarias no obstructivas; SCA: Síndrome coronario agudo; ICP: Intervencionismo coronario percutáneo (Angioplastia- Stent); EAC: Enfermedad arterial coronaria.

Tomado de: Beltrame J. y col., 2017 (20).

el estudio de Isogai y col., con 21.512 pacientes, en el cual se utilizó para las PPVCG tanto la acetilcolina como la ergonovina, se evidenció que la tasa de complicaciones mayores definidas para el estudio, como necesidad de desfibrilación, maniobras de resucitación cardiopulmonar, dispositivo de asistencia circulatoria y muerte, fue de $0,7 \%$ en total, $0,9 \%$ con la acetilcolina y $0,4 \%$ con la ergonovina (22). En un meta-análisis de más de 20 estudios sobre la seguridad de las PPVCC mostraron una baja incidencia de complicaciones mayores, en total $0,89 \%$ con una mortalidad de $0,006 \%$, por lo que consideraron que las PPVCC no presentan un riesgo adicional a la realización de la angiografía coronaria (23). Existe resistencia en muchos cardiólogos intervencionistas al uso de las PPVCG, en parte porque existe una limitada experiencia en su uso y debido al hecho de que, al no haber sido aprobadas por la FDA, conlleva implicaciones legales y de reintegro por los seguros, es por eso que, su uso en EE. UU. se restringe a protocolos de investigación en centros hospitalarios universitarios (24). Algunos investigadores alegan que se requieren ensayos clínicos internacionales que comparen el mismo protocolo de PPVCG entre diferentes países, esperando así que, según los resultados, las guías: americana y europea $(24,25)$ cuya indicación es clase IIa, le den a las PPVCC para la evaluación de la AVE indicación clase I, similar a las guías de la Sociedad Japonesa de Circulación (23, 25). Esta es una de las razones por las cuales, en muchos centros, ante un paciente con INOCA, se asume erróneamente un origen microvascular, al no descartar el vasoespasmo coronario epicárdico.

El diagnnóstico de la AVE también puede ser apoyado por el uso del Holter en busca de cambios dinámicos del segmento ST-T su- 
gestivos de isquemia, el cual es clase IIa según la guía europea de enfermedad arterial coronaria estable del 2013 (25). Otras técnicas diaǵnósticas que se han evaluado son la prueba de hiperventilación y la de estimulación cutánea con frio (cold pressor test) durante la anǵiografía, pero estas son muy limitadas por su baja sensibilidad y por eso no se recomiendan (25).

El síndrome INOCA: Isquemia miocárdica y angina con coronarias no obstructivas

Una vez descartadas todas las causas descritas en el algoritmo de la Figura 1, el síndrome INOCA constituye un grupo heterogéneo de pacientes con angina de etiologías no fácilmente detectables y con múltiples mecanismos fisiopatológicos posibles, dentro de las cuales están: 1) Enfermedad aterosclerótica oculta de vasos mayores, 2) Alteraciones de la microcirculación (vasos con menos de $500 \mu \mathrm{m}$ de diámetro), causadas por espasmo microvascular, vasoconstricción prearteriolar en parches, enfermedad de las pequeñas arterias y trastornos en la capacidad de vasodilatación de la microcirculación 3) Vasoespasmo de arterias coronarias epicárdicas, 4) Trastornos neuronales de la percepción del dolor visceral: síndrome del corazón sensible $(6,14)$.

\section{MECANISMOS FISIOPATOLÓGICOS}

\section{Disfunción coronaria microvascular}

El sistema arterial coronario está compuesto de tres compartimientos con diferentes funciones: El compartimiento proximal, formado por las grandes arterias epicárdicas que tienen función de capacitancia y ofrecen poca resistencia al flujo coronario y sus diámetros van desde $500 \mu \mathrm{m}$ hasta 4 a $5 \mathrm{~mm}$; el compartimiento intermedio está representado por las prearteriolas y se caracteriza por presentar en su longitud una caída progresiva de la presión, sus diámetros están entre $100 \mu \mathrm{m}$ a $500 \mu \mathrm{m}$ y su función es mantener la presión de perfusión dentro de un rango estrecho hasta el origen de las arteriolas, en presencia de cambios de flujo y de presión intravascular. El compartimiento distal está representado por las arteriolas intramurales, las cuales no son visualizadas en la angiografía; representan la microcirculación y se caracterizan por presentar una caída considerable de la presión coronaria con importante disminución de la resistencia vascular como respuesta a los metabolitos miocárdicos difusibles; su función es mantener el balance entre la oferta del flujo sanguíneo miocárdico y el consumo de oxígeno (27). En 1985 Cannon y Epstein, introdujeron el término "angina microvascular" en relación al aumento en la sensibilidad de la microcirculación coronaria, ante estímulos de vasoconstricción asociados a la disminución en la capacidad de vasodilatación. Ellos propusieron que la causa patogénica de este síndrome, se debía a la disfunción de las pequeñas arteriolas intramurales (28). La disfunción microvascular (DMV) está definida como la disfunción endotelial y de la pared vascular epicárdica y microvascular, que limita la perfusión miocárdica, más frecuentemente detectada por la reserva de flujo coronario global $(6,9)$, (Tabla II).

A partir del 2007 la DMV se clasificó en 4 tipos de acuerdo con las características clínicas con que ocurre: Tipo 1) DMV en ausencia de enfermedad miocárdica y enfermedad arterial coronaria obstructiva (es la que ocupa este articulo); tipo 2) DMV en presencia de enfermedad miocárdica; tipo 3) DMV en presencia de enfermedad arterial coronaria obstructiva y tipo 4) DMV iatrogénica, posterior a angioplastia o a cirugía de revascularización miocárdica (27) (Tabla III).

Los estudios con tomografía de emisión de positrones (PET: Positron Emission Tomography), permiten cuantificar con exactitud, volúmenes en tejidos, por lo que han logrado establecer el rango normal del flujo sanguíneo miocárdico absoluto (FSMA, ml/ $\mathrm{min} / \mathrm{g}$ ), y la reserva de flujo coronario global (CFR), que es la relación entre el FSMA en vasodilatación máxima con adenosina, dividida entre el FSMA basal (13). La evidencia de la participación del compartimiento

Vol. 61(4): 376 - 392, 2020 


\section{TABLA II \\ CAUSAS DE DISFUNCIÓN MICROVASCULAR.}

1 Estrés oxidativo y proceso inflamatorio vascular por factores de riesgo cardiovascular y noxas externas.

2 Disminución de la reserva e flujo coronario (CFR) por alteración de la capacidad vasodilatadora y aumento de la resistencia al flujo sanguíneo coronario.

3 Mecanismos dependientes del endotelio microvascular, con falla en el aumento del flujo sanguíneo coronario con la prueba de acetilcolina o cold stressor test, que miden la disminución o falta de liberación de óxido nítrico del endotelio.

4 Mecanismos independientes del endotelio. El espasmo microvascular coronario es bloqueado por el fusidil, un inhibidor de la Rho-Kinasa, que participa en la vía intracelular de contracción del músculo liso vascular, independiente del endotelio. La adenosina produce vasodilatación de la microcirculación miocárdica, no dependiente del endotelio.

Tomado de: Novo G, y col. 2014 (9) y Susuki X. 2015 (29).

TABLA III

GLASIFICACIÓN DE LA DISFUNCIÓN MICROVASGULAR CORONARIA.

\begin{tabular}{|c|c|c|c|}
\hline Clasificación & Condición & Escenario Clínico & Mecanismos Patogénicos \\
\hline Tipo 1 & $\begin{array}{l}\text { Sin EAC obstructiva ni } \\
\text { enfermedad miocárdica }\end{array}$ & $\begin{array}{l}\text { Factores de Riesgo CVS } \\
\text { Angina Microvascular }\end{array}$ & $\begin{array}{l}\text { Disfunción Endotelial } \\
\text { Disfunción del MLV } \\
\text { Remodelado vascular. }\end{array}$ \\
\hline Tipo 2 & $\begin{array}{l}\text { Con enfermedad } \\
\text { miocárdica }\end{array}$ & $\begin{array}{l}\text { C. Dilatada } \\
\text { C. Hipertrófica } \\
\text { Miocarditis } \\
\text { Enf. del Miocardio } \\
\text { Obst. del TSVI }\end{array}$ & $\begin{array}{l}\text { Remodelado vascular } \\
\text { Disfunción del MLV } \\
\text { Compresión extramural } \\
\text { Obstrucción luminal }\end{array}$ \\
\hline Tipo 3 & Con EAC obstructiva & $\begin{array}{l}\text { EAC estable } \\
\text { Síndrome Coronario } \\
\text { Agudo }\end{array}$ & $\begin{array}{l}\text { Disfunción Endotelial } \\
\text { Disfunción del MLV } \\
\text { Obstrucción luminal }\end{array}$ \\
\hline Tipo 4 & Iatrogénica & $\begin{array}{l}\text { ICP o Cirugía de } \\
\text { Revascularización } \\
\text { miocárdica }\end{array}$ & $\begin{array}{l}\text { Disfunción Endotelial } \\
\text { Disfunción autonómica } \\
\text { Obstrucción luminal }\end{array}$ \\
\hline
\end{tabular}

EAC: Enfermedad Arterial Coronaria; CVS: Cardiovascular; MLV: Musculo Liso Vascular; C: Cardiopatía;

TSVI: Tracto de salida del ventrículo izquierdo; ICP: Intervencionismo Coronario Percutáneo.

Tomado de: Crea F. y col. 2014 (13) y Camici P. y col. 2007 (27).

microvascular como causa de la AMV, se ha obtenido de múltiples estudios de pacientes que presentaban angina, con pruebas de inducción de isquemia (ecocardiograma estrés y/o cintilografía de perfusión miocárdica), con angiografía coronaria sin lesiones obstructivas y estudios de provocación de vasoconstricción con acetilcolina o ergonovina, que no presentaban vasoespasmo de coro- narias epicárdicas en la anǵiografía, comparados con sujetos controles sin síntomas ni alteraciones sugestivas de isquemia en el ECG de esfuerzo, con estudios de perfusión y angiografías normales. Las PPVCC para la evaluación de la DMV, tienen indicación clase IIb, en la guía europea de enfermedad arterial coronaria estable, en pacientes con angiografía que muestra coronarias sin lesio- 
nes $(16,28)$. Además, en estos pacientes con angina microvascular, cuando son sometidos a estrés, muchos estudios han demostrado alteraciones del metabolismo miocárdico y alteraciones de la CFR (Tabla II). En aquellos pacientes sometidos a biopsias miocárdicas, se demostró alta prevalencia de alteraciones estructurales de los vasos de la microcirculación con reducción de la luz, debido a hiperplasia fibro-intimal, fibrosis intersticial y perivascular, ausentes en el grupo control $(14,16,29)$.

La determinación de la CFR con PET ha aportado los datos más sustanciales con relación a la DMV y el riesǵo de desenlaces adversos, entre los pacientes con AMV, aquellos con GFR reducida $(<2,0)$, la tasa de eventos cardiovasculares mayores (ECM) dada por infarto de miocardio, revascularización, hospitalización por insuficiencia cardiaca o muerte, aumentó 2,5 veces en 3 años, frente a aquellos con CFR normal. Mientras que las mujeres representan un $70 \%$ de la prevalencia de INOCA, estos estudios mostraron que el riesgoo aumentado por la limitación de la CFR cuando se presenta, no parece ser diferente entre el hombre y la mujer (5)(6). Es fundamental distinguir la AMV primaria sin enfermedad cardiaca subyacente; que es la que pertenece a la INOCA (SXC), al descartar las patologías cardíacas que cursan con AMV secundaria, tales como diferentes causas de enfermedad miocárdica, la miocardiopatía hipertrófica, la enfermedad coronaria obstructiva ateroselerótica y la enfermedad valvular $(6,9,14)$.

\section{Enfermedad aterosclerótica no obstructiva}

En la década de 1980, Glagov y col. describieron el remodelado vascular producido por la aterosclerosis, donde las placas de ateroma que ocupan la íntima y la capa media vascular pueden crecer en un espacio importante en el vaso, con remodelado expansivo compensatorio que se ubica en la pared arterial hacia el exterior, con poca o nula expresión en la luz del segmento arterial afectado, sin producir estenosis. Muchas de estas placas, conocidas como vulnerables, se complican con ruptura del ateroma y evolucionan con aterotrombosis, desencadenando ya sea un síndrome coronario agudo, ictus o muerte súbita, según el territorio afectado. Por otro lado, el remodelado constrictivo es aquel que se produce cuando la placa (generalmente fibrosa), crece hacia la luz del vaso, produciendo estenosis en forma progresiva; esta forma se observa más frecuentemente en la angina estable (30).

Cerca del 30\% de los pacientes diabéticos puede presentar enfermedad coronaria difusa que, en la angiografía, en muchos casos, se puede visualizar como coronarias sanas, lisas y delgadas. Algunos signos angiográficos de enfermedad difusa son: las irregularidades parietales en sierra dentada, presencia del diámetro de un segmento distal mayor que el proximal o segmentos medios y distales muy delgados. El IVUS nos permite observar claramente el ateroma con distribución difusa, concéntrica y uniforme en la pared del vaso, algunas de estas placas presentan remodelado expansivo que no protruyen en la luz, por lo que no se observan en la angiografía coronaria (31).

Existen autores que postulan que la enfermedad microvascular coronaria no es más que una expresión precoz de la enfermedad ateroselerótica incipiente, con disfunción endotelial local y sistémica, entre ellos Weidermann y col., quienes en 1995 (32), estudiaron pacientes con SXC, utilizando IVUS; describieron 3 patrones: Patrón 1, normal sin placas ateromatosas; patrón 2 , con placas ateromatosas pequeñas o moderadas, con remodelado expansivo, sin reducción de la luz; patrón 3, con aumento del grosor de la íntima y la media. Los estudios de inducción de isquemia con medición de los diámetros de las arterias epicárdicas con IVUS, mostraron en el patrón 1 , vasodilatación de coronarias epicárdicas y en los patrones 2 y 3 vasoconstricción, lo cual reflejaba disfunción endotelial.

La noción de que las mujeres cuyas angiografías resultan normales y su pronóstico 
es benigno, debería ser reevaluada, a la luz de los resultados del estudio WISE, el cual evidenció que el $80 \%$ de estas pacientes, estudiadas con IVUS, tenía aterosclerosis coronaria no obstructiva por remodelado vaseular expansivo $(3,25)$.

Es importante diferenciar entre pacientes con enfermedad arterial coronaria no obstructiva, cuya angiografía coronaria muestra irregularidades parietales, con cerca de 30\% de reducción de la luz y/o lesiones intermedias con estenosis de 40 a 50\%, y pacientes sin evidencia de enfermedad arterial coronaria, con angiografía de aspecto anatómico completamente normal. En los primeros existe un incremento de la mortalidad cardiovascular al seguimiento de 5 años $\mathrm{y}$ en aquellos con angiografías normales hay un incremento de eventos, pero no de mortalidad (6).

\section{Hipótesis del corazón sensible: Etiología no isquémica}

Esta hipótesis deriva de las observaciones iniciales y los estudios donde se demostró que en más de la mitad de los pacientes con SXC, se podía inducir dolor característico anginoso, con la estimulación eléctrica ventricular derecha con un marcapaso, con la inyección de contraste en la coronaria izquierda y en cerca del 10 a $15 \%$, con el uso de adenosina o dipiridamol intracoronarios; por otro lado, rara vez se induce dolor con estos estímulos en los pacientes con enfermedad arterial coronaria obstructiva o enfermedad valvular (28). La adenosina es uno de los mediadores moleculares del dolor cardiaco y sistémico, que actúa mediante la activación de vías aferentes simpáticas ventriculares, que envían las señales hacia la médula, tálamo y corteza cerebral. Esto sugiere que parte del mecanismo de producción del dolor en algunos pacientes, pudiera estar relacionado con falla en el metabolismo de la adenosina tisular local, creando su acúmulo y desencadenando la estimulación de las vías del dolor en ausencia de isquemia (33-35). Es aceptado que la activación de receptores de adenosina del tipo $\mathrm{A}_{1}$ tiene efectos anti-nociceptivos, mientras que los del tipo $\mathrm{A}_{2 \mathrm{~A}}$, muestran efectos pro-nociceptivos espinales; esto a nivel del tejido miocárdico, sugiere la posible existencia de un desbalance entre los efectos de la adenosina sobre estos receptores (36). En cuanto a las vías neuronales relacionadas con la percepción de anǵina, existen estudios en pacientes con SXC, comparados con sujetos sanos, evaluados con ecocardiografía de estrés con dobutamina y análisis de perfusión cerebral con PET, como índice de actividad neuronal en reposo y en estrés; la dobutamina produjo angina severa con infra-desnivel del segmento ST en el ECG y contractilidad ventricular izquierda normal en todos los pacientes con SXC, mientras que en los sujetos controles no hubo síntomas, ni alteraciones en el ECG, ni en la motilidad ventricular. En cuanto a la perfusión cerebral en los pacientes con SXC y no en los sujetos controles hubo un incremento en la perfusión en el área de la ínsula de la corteza cerebral del hemisferio derecho. Bajo esta óptica de la hipótesis del corazón sensible, alguunos pacientes con SXC podrían tener un patrón alterado de la activación cortical cerebral de las señales aferentes viscerales, que pudieran contribuir a la percepción anormal del dolor durante estrés cardiaco, en ausencia de isquemia miocárdica $(34,37)$.

\section{DIAGNÓSTICO ESPECÍFICO}

En la práctica clínica estándar, el uso de las pruebas funcionales coronarias (PPVCC, CFR, FFR) ad hoc, en los pacientes con INOCA, está actualmente restringido a unos pocos centros de interés académico; esto en parte está relacionado con la falta de evidencia, de que esta evaluación en busca del diaǵnóstico especifico, tenǵa alguuna utilidad clínica o produzea mejoría en el bienestar general del paciente $(6,21)$. Debido a la multiplicidad de hipótesis para explicar una misma manifestación clínica y lo complejo del tema, en cuanto a su evaluación, desde 
el año 2012 se conformó un ǵrupo de investigadores para unificar criterios y estandarizar el diagnóstico de lo que se denominó "angina microvascular", que sirve como marco de referencia en la práctica clínica y para la investigación (12).

\section{Evaluación de la microcirculación}

El compartimiento microvascular puede ser evaluado con pruebas no invasivas e invasivas. Las no invasivas son la determinación de la CFR con PET y la Resonancia Magnética Cardíaca (RMC), que determinan la perfusión miocárdica en reposo y con estrés físico, o vasodilatación farmacológica de la microcirculación con adenosina. Las pruebas invasivas son la angiografía coronaria con provocación de vasoespasmo mediante el uso de acetilcolina o erǵonovina; si el sujeto es sano habrá vasodilatación, si tiene disfunción endotelial en una o varias coronarias epicárdicas, estas responderán con vasoespasmo y si hay DMV habrá angina con alteraciones en el ECG sugestivas de isquemia, vasoconstricción microvascular con reducción de las velocidades de flujo y aumen- to de la resistencia vascular. Esta respuesta fisiopatológica que no puede ser visualizada angiográficamente, se evalúa con una guía Doppler intracoronaria que mide velocidad de flujo, o con una guía de termodilución que mide directamente el flujo coronario y la resistencia microvascular $(12,38)$. Para considerar la sospecha de angina microvascular deben cumplirse 4 criterios básicos, que son: 1) Presencia de angina de pecho o su equivalente, 2) Ausencia de enfermedad coronaria obstructiva demostrada por angiografía o angioTAC, 3) Evidencia objetiva de angina microvascular (angina con ecocardiograma stress negativo) y 4) Evidencia de alteración funcional de la microvasculatura coronaria (Tabla IV) $(6,12,13)$.

Recientemente se han desarrollado métodos de medición del flujo coronario angiográfico "off-line", sin la necesidad de usar instrumentación endovascular, sin embargoo, esta tecnología con futuro promisorio todavía no ha sido validada en el síndrome INOCA $(39,40)$. En el reciente estudio CorMica, específico sobre el síndrome INOCA, cuando se realizó la evaluación funcional coronaria

TABLA IV

CRITERIOS CLÍNICOS DE SOSPECHA DE ANGINA MICROVASCULAR.

1.- SÍNTOMAS DE ISQUEMIA MIOCÁRDICA

a) Angina de esfuerzo y /o reposo

b) Angina equivalente (Disnea o Arritmia)

2.- AUSENCIA DE ENFERMEDAD ARTERIAL GORONARIA OBSTRUCTIVA

a) Angiografía coronaria $(<50 \%$ de estenosis o FFR $>0,80)$

b) Angio-tomografía coronaria con coronarias sin lesiones obstructivas

3.- EVIDENCIA OBJETIVA DE ANGINA MICROVASCULAR

a) Cambios isquémicos en el ECG durante el episodio de dolor torácico

b) Prueba de inducción de isquemia: Perfusión radioisotópica, Eco-stress

4.- EVIDENCIA DE ALTERAGIÓN EN LA FUNCIÓN MICROVASGULAR CORONARIA

a) Alteración de la Reserva del Flujo Coronario global (CFR <2,0)

b) Espasmo Microvascular Coronario (angina y cambios isquémicos en el ECG, sin espasmo coronario epicárdico con la prueba de acetilcolina).

c) Índice de resistencia microvascular coronario elevado (IRM $>25$ )

d) Fenómeno de flujo lento coronario (Conteo de Cuadros Correǵidos TIMI > 25)

Anǵina Microvascular Definitiva: Si se cumplen los 4 criterios

Angina Microvascular Sospechosa: Criterios 1 y 2, más criterio 3b o 4b.

Tomado de: Bairey Merz CN y col., 2017 (6), y Ong P y col 2018 (12).

Vol. 61(4): 376 - 392, 2020 
(con CFR, FFR y PPVCC con acetilcolina), se observó que la prevalencia de los diaǵnósticos era la siguiente: AMV 52\%, AVE en 17\%, la angina mixta (AMV y AVE) 20\% y el dolor de pecho no isquémico 11\% (21).

\section{CONSIDERACIONES FINALES}

El diagnóstico de INOCA generalmente se orienta hacia la AMV, sin embargo, actualmente en muchos centros, a los pacientes con síntomas y pruebas no invasivas de inducción de isquemia miocárdica positiva, con angiografía coronaria de aspecto normal, se les hace el diagnóstico de INOCA sin haber descartado el vasoespasmo coronario epicárdico con PPVCG, ni haber realizados estudios de CFR para definir si el problema es microvascular (6), lo que pudiera estar ocasionando un sobrediaǵnóstico de la AMV, con subvaloración de la AVE, lo que resulta en un manejo terapéutico inadecuado. Esta pudiera ser una de las razones del porqué en el reciente meta-análisis de Radico y col., la INOCA sigue siendo considerada en su comportamiento clínico, como heterogénea y de difícil manejo, al incluir entidades con diferentes mecanismos fisiopatológicos (41). Los investigadores que estudian este síndrome consideran que es necesaria la realización de más estudios aleatorizados, con diseños adecuados, para así lograr un protocolo universal de diagnnóstico. El estudio británico CorMica, al igual que la experiencia japonesa, ratifican la seguridad de las PPVCG para evaluar la AVE y la AMV, por lo que sugieren la reevaluación de estas pruebas con estudios de mejor diseño y a mayor escala en EE. UU. y otros centros europeos, con la finalidad de obtener mayor nivel de evidencia que pudiera permitir su aprobación por los entes regulatorios. Sería interesante también profundizar en el estudio de la hipótesis nociceptiva (corazón sensible) y ver qué posición tendría esta, en la evaluación del síndrome INOCA $(23,24,26)$.

\section{MANEJO TERAPÉUTICO}

Hasta ahora las estrategias del manejo del síndrome INOCA, no se basan en pautas o guías de tratamiento, ya que no existe suficiente evidencia con ensayos aleatorizados, específicos para esta entidad, que sirvan de apoyo para su elaboración (6). El objetivo fundamental del tratamiento es el control de los síntomas y de la morbilidad (28). Las recomendaciones terapéuticas se basan en el abordaje de los principales mecanismos fisiopatológicos involucrados. La evidencia actual disponible, sugiere el beneficio potencial en todos los pacientes con INOCA, de la estrategia antiaterogénica, que aborda la disfunción endotelial e inflamación vascular: Esta incluye el uso de estatinas, inhibidores de la enzima convertidora de angiotensina, bloqueadores de los receptores de angiotensina y antiagregantes (42-44). Cuando se diaǵnostica la AVE, se aplica la estrategia para vasoespasmo y anti-isquémica: Nitratos, bloqueadores de los canales de calcio y en los casos más resistentes se asocia nicorandil y/o ranolazina, y se sugiere evitar los beta bloqueadores particularmente los no cardioselectivos $(26,43,45)$. Con la AMV, se desaconseja el uso de nitratos, puesto que los estudios no han mostrado beneficio y en algunos casos pudieran empeorar los síntomas $(21,44,46)$. Se recomiendan como anti-isquémicos, los beta-bloqueadores cardioselectivos (bisoprolol y nebivolol) o aquellos con bloqueo alfa1 tipo carvedilol y los bloqueadores de los canales de calcio, y cuando hay pobre respuesta, se puede asociar la ranolazina $(26,47,48)$. En los pacientes con angina de causa no isquémica, se sugiere la estrategia anti-nociceptiva: La aminofilina como inhibidor selectivo del receptor P1 de la adenosina, que ha mostrado mejoría de síntomas y de la capacidad de ejercicio en estudios con muestras limitadas $(35,47)$. El uso de antidepresivos tricíclicos como la Imipramina a bajas dosis, con me- 
joría en algunos pacientes, pudiera ser útil, pero está limitado por sus efectos adversos y porque la evidencia es escasa (48). Al establecerse el diagnóstico de AMV, la estrategia anti-aterogénica y antinflamatoria vascular, han demostrado beneficio con mejoría de la DMV evaluada con CFR (43). El manejo y control de los factores de riesgo cardiovasculares son fundamentales y los programas de ejercicio con rehabilitación cardíaca además de mejoría de los síntomas, han demostrado efecto beneficioso en la microcirculación, con mejoría en la CFR evaluada con RMC.

Existen otras modalidades como las terapias psicológicas cognitivas conductuales, la meditación y el uso de la estimulación nerviosa eléctrica de la medula espinal, con escasos ensayos en muestras limitadas y algunos con resultados contradictorios que no permiten una recomendación formal. Se requieren estudios prospectivos con muestras adecuadas para evaluar la capacidad que pudieran tener diferentes estrategias terapéuticas de modificar la CFR $(6,41,47,48)$.

Esta revisión resume la literatura actual en cuanto a la definición del síndrome INOCA y enfoca la necesidad de la detección de su mecanismo fisiopatológico específico, haciendo énfasis en los esfuerzos de investigaciones actuales para establecer criterios diaǵnósticos claros, basados en evidencias $(6,12$, $26,41)$. Se abordaron la experiencia y los estudios de las pruebas de provocación de vasoconstricción y la determinación de la CFR en estos pacientes. Si bien la terapia guiada por un diagnóstico preciso de angina vasoespástica o microvascular puede ayudar en el control del dolor, no existen ensayos clínicos aleatorizados con potencia suficiente hasta la fecha, que comparen la mortalidad y la calidad de vida, entre el tratamiento empírico versus el tratamiento guiado por pruebas funcionales, que soporten el uso rutinario de estas pruebas. Existen proyectos en este sentido, como el CorMica, que buscan establecer el enlace entre el mecanismo fisiopatológico especifico y el desenlace clínico $(13,19,26)$. El tratamiento de esta entidad actualmente debe ser individualizado y basarse en el abordaje, inicialmente de manera empírica, considerando los múltiples mecanismos etiológicos posibles, mientras se completa el protocolo diaǵnóstico y se define su causa específica, con el objetivo del control y mejoría de síntomas y su pronóstico $(6,42)$.

\section{REFERENCIAS}

1. Johnson LW, Krone R. Cardiac eatheterization 1991: A report of the registry of the society for cardiac angiography and interventions (SCA\&I). Cathet Cardiovase Diagn 1993;28(3):219-220.

2. Patel MR, Peterson ED, Dai D, Brennan JM, Redberg RF, Anderson HV, Brindis RG, Douglas PS. Low diagnostic yield of elective coronary angiography. $\mathrm{N}$ Engl $\mathrm{J}$ Med 2010;362(10):886-895. http://wrwr.nejm. org/doi/abs/10.1056/NEJMoa0907272.

3. Gulati M, Shaw LJ, Bairey Merz CN. Myocardial ischemia in women: Lessons from the NHLBI WISE study. Clin Cardiol 2012;35(3):141-148.

4. López-Hidalgo M, Rivas CC, Eblen-Zajjur A. Síndrome coronario agudo con coronarias sin lesiones obstructivas. Rev Fed Arg Cardiol 2018;47(3):140-146. http:// wrww.fac.org.ar/2/revista/18v47n3/original/05/hidalgo.pdf

5. Jespersen L, Hvelplund A, Abildstrøm SZ, Pedersen F, Galatius S, Madsen JK, Jørgensen E, Kelbak H, Prescott E. Stable angina pectoris with no obstructive coronary artery disease is associated with increased risks of major adverse cardiovascular events. Eur Heart J 2012;33(6):734-744.

6. Merz CNB, Pepine CJ, Walsh MN, Fleg JL. Ischemia and No Obstructive Coronary Artery disease (INOCA): Developing evidence-based therapies and research agenda for the next decade. Circulation 2017;135(11):1075-1092.

7. Arbogast R, Bourassa MG. Myocardial function during atrial pacing in patients with angina pectoris and normal coronary arteriograms. Am J Cardiol 1973;32(3):257-263. doi: 10.1016/s00029149(73)80130-4.

8. Kemp HG, Vokonas PS, Cohn PF, Gorlin R. The anginal syndrome associated 
with normal coronary arteriograms. Report of a six year experience. Am J Med 1973;54(6):735-742.

9. Novo G, Novo S. Coronary microvascular dysfunction: An update. e-journal of the ESC Council for Cardiology Practice 2014; 13 (5).

10. Merz CNB, Kelsey SF, Pepine CJ, Reichek N, Reis SE, Rogers WJ, Sharaf BL, FACC, Sopko G. The Women's Ischemia Syndrome Evaluation (WISE) study: Protocol design, methodology and feasibility report. J Am Coll Cardiol 1999;33(6):1453-1461. http://dx.doi.org/10.1016/S07351097(99)00082-0

11. Agrawal S, Mehta PK, Bairey Merz CN. Cardiac Syndrome X. Cardiol Clin 2014 Aug;32(3):463-478. doi:10.1016/j.ccl.2014. 04.006.

12. Ong P, Camici PG, Beltrame JF, Crea F, Shimokawa H, Sechtem U, Kaski JC, Bairey Merz CN. International standardization of diaǵnostic criteria for microvascular angina. Int J Cardiol 2018;250:16-20.

13. Crea F, Camici PG, Bairey Merz CN. Coronary microvascular dysfunction: An update. Eur Heart J 2014;35(17):1101-1111.

14. Schwartz L, Bourassa MG. Evaluation of patients with chest pain and normal coronary angiograms. Arch Intern Med 2001;161(15):1825-1840. doi: 10.1001/archinte.161.15.1825.

15. Kang SJ, Lee JY, Ahn JM, Song HG, Kim WJ, Park DW, Yun S-C, Lee S-W, Kim Y-H, Mintz GS, Lee CW, Park S-W, Park SJ. Intravascular ultrasound-derived predictors for fractional flow reserve in intermediate left main disease. JACC Cardiovase Interv 2011;4(11):1168-1174. http://dx.doi.org/ 10.1016/j.jcin.2011.08.009

16. Jasti V, Ivan E, Yalamanchili V, Wongpraparut N, Leesar MA. Correlations between fractional flow reserve and intravascular ultrasound in patients with an ambiguous left main coronary artery stenosis. Circulation 2004;110(18):2831-2836.

17. Nørgaard BL, Terkelsen CJ, Mathiassen ON, Grove EL, Bøtker HE, Parner E, Leipsic J, Steffensen FH, Riis AH, Pedersen K, Christiansen EH, Mæng M, Krusell LR, Kristensen SD, Eftekhari A, jacobsen L, Jensen JM. Coronary CT Anǵioǵraphic and flow reserve-guided management of pa- tients with stable ischemic heart disease. $\mathrm{J}$ Am Coll Cardiol 2018;72(18):2123-2134.

18. Kaski JC. Sindrome X: El dilema de la anǵina de pecho con coronarias angioǵraficamente normales. Rev Arǵent Cardiol 1993;61(4):327-332.

19. Ong $P$, Athanasiadis $A$, Sechtem U. Intracoronary acetylcholine provocation testing for assessment of coronary vasomotor disorders. J Vis Exp 2016;2016(114):1-8.

20. Beltrame JF, Crea F, Kaski JC, Ogawa H, Ong P, Sechtem U, Shimokawa H, Bairey Merz CN. International standardization of diaǵnostic criteria for vasospastic angina. Eur Heart J 2017;38(33):2565-2568.

21. Ford TJ, Corcoran D, Oldroyd KG, MeEntegart M, Rocchiccioli P, Watkins S, Brooksbank K, Padmanabhan S, Sattar N, Briggs A, McConnachie A, Touyz R, Berry C. Rationale and design of the British Heart Foundation (BHF) Coronary Microvascular Angina (CorMicA) stratified medicine clinical trial. Am Heart J 2018;201:86-94. https://doi.org/10.1016/j.ahj.2018.03.010.

22. Isogai T, Yasunaga H, Matsui H, Tanaka H, Ueda T, Horiguchi H, Fushimi K. Serious cardiac complications in coronary spasm provocation tests using acetylcholine or ergonovine: Analysis of 21512 patients from the diagnnosis procedure combination database in Japan. Clin Cardiol 2015;38(3):171-177.

23. Sueda S, Kohno H. Overview of complications during pharmacological spasm provocation tests. J Cardiol 2016;68(1):1-6. http://dx.doi. org/10.1016/j.jicc.2016.03.005.

24. Zaya M, Mehta PK, Bairey Merz CN. Provocative testing for coronary reactivity and spasm. J Am Coll Cardiol 2014;63(2):103109. DOI: $10.1016 /$ j.jacc.2013.10.038

25. Montalescot G, Sechtem U, Achenbach S, Andreotti F, Arden C, Budaj A, Bugiardini R, Crea F, Cuisset T, Di Mario C, Ferreira JR, Gersh BJ, Gitt AK, Hulot J-S, Marx N, Opie LH, Pfisterer M, Prescott E, Ruschitzka F, Sabate' M, Senior R, Taggart DP, Van derWall EE, Vrints CJM. 2013 ESC guidelines on the management of stable coronary artery disease. Eur Heart J 2013;34(38):2949-3003.

26. Ford TJ, Berry C. How to diagnnose and manage angina without obstructive coro- 
nary artery disease: Lessons from the british heart foundation CorMicA Trial. Interv Cardiol Reve 2019;14(2):76. https://doi. org/10.15420/icr.2019.04.R1

27. Camici PG, Crea F. Coronary microvascular dysfunction. N Enǵl J Med 2007;356:830_ 840 .

28. Cannon RO. Microvascular angina and the continuing dilemma of chest pain with normal coronary angiograms. J Am Coll Cardiol 2009;54(10):877-885.

29. Suzuki H. Different definition of microvascular angina Eur J Clin Invest 2015; 45(12):1360-1366.

30. Glagov S, Weisenberg E, Zarins CK, Stankunavicus R, Colettis GJ. Compensatory enlargement of human atherosclerotic coronary arteries. N Enǵl J Med 1987;316:1371-1375.

31. Reynolds HR, Srichai MB, Iqbal SN, Slater JN, Mancini GBJ, Feit F, Pena-Sing I, Axel L, Attubato MJ, Yatskar L, Kalhorn RT, Wood DA, Lobach IV, Hochman JS. Mechanisms of myocardial infarction in women without angiographically obstructive coronary artery disease. Circulation 2011;124(13):1414-1425.

32. Wiedermann JG, Schwartz A, Apfelbaum M. Anatomic and physiologic heterogeneity in patients with syndrome X: An intravascular ultrasound study. J Am Coll Cardiol 1995;25(6):1310-1317.

33. Eblen-Zajjur A. Neurofisiología de la nocicepción. Gac Med Caracas 2005 [cited 2018 Sep 7];113(4):466-73. http://www.scielo.org.ve/ scielo.php?script $=$ sci_arttext\&pid $=\mathrm{S} 0367$ 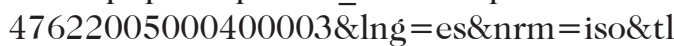 ng $=\mathrm{es}$

34. Willis, WD, Coggeshall RE. Sensory mechanisms of the spinal cord. 3rd ed. Media S Science Business, editor. New York: Klwer Academic/Plenum Publisher; 2004. 612618 p.

35. Crea F, Pupita G, Galassi AR, El-Tamimi H, Kaski JC, Davies G,? Maseri A. Role of adenosine in pathogenesis of anginal pain. Circulation. 1990;81(1):164-172.

36. Sawynok J, Liu XJ. Adenosine in the spinal cord and periphery: Release and regulation of pain. Prog Neurobiol 2003;69(5):313-340.

37. Rosen SD, Paulesu E, Wise RJS, Camiei PG. Central neural contribution to the perception of chest pain in cardiac syndrome X. Heart 2002;87(6):513-519. doi: 10.1136/heart.87.6.513.

38. Veer M Van, Adjedj J, Wijnbergen I, Nunen LX Van, Pijls NHJ, De Bruyne B. Novel monorail infusion catheter for volumetric coronary blood flow measurement in humans: in vitro validation. EuroIntervention 2016; 12(6):701-707. doi: 10.4244/EIJV12I6A114.

39. Pellicano M, Lavi I, De Bruyne B, VakninAssa H, Assali A, Valtzer O, Lotringer Y, Weisz G, Almagor Y, Xaplanteris P, Kirtane AJ, Codner p, Leon MB, Kornowski R. Validation study of image-based fractional flow reserve during coronary angiography. Cire Cardiovase Interv 2017; 10(9):e005259. doi: 10.1161/CIRCINTERVENTIONS.116.005259.PMID: 28916602

40. López-Hidalgo M. Caracterización mutiparamétrica del flujo sanguíneo coronario mediante densimetría de contraste en angioǵrafia coronaria convencional de pacientes sin lesiones anǵiograficas [Tesis Doctoral] Valencia: Universidad de Carabobo, Venezuela, 2020.

41. Radico F, Zimarino M, Fulǵenzi F, Ricei F, Di Nicola M, Jespersen L, Chang SM, Humphries KH, Marzilli M, De Caterina R. Determinants of long-term clinical outcomes in patients with angina but without obstructive coronary artery disease: a systematic review and meta-analysis. Eur Heart J 2018; 39(23):2135-2146. doi: 10.1093/ eurheartj/ehy185.

42. Ong $\mathbf{P}$, Athanasiadis A, Sechtem U. Pharmacotherapy for coronary microvascular dysfunction. Eur Heart J Cardiovase Pharmacother 2015;1(1):65-71. doi: 10.1093/ ehjcvp/pvu020.

43. Caliskan M, Erdogan D, Gullu H, Topeu S, Ciftei O, Yildirir A MH. Effects of a atorvastatin on coronary flow reserve in slow coronary flow. Clin Cardiol 2007;30(7):475479. doi: 10.1002/clc.20140.

44. Turgeon RD, Pearson GJ, Graham MM. Pharmacologic treatment of patients with myocardial Ischemia with no obstructive coronary artery disease. Am J Cardiol 2018;121(7):888-895. https://doi. org/10.1016/j.amjcard.2017.12.025.

45. Merz CNB, Handberg EM, Shufelt CL, Mehta PK, Minissian MB, Wei J, Thom- 
son LEJ, Berman DS, Shaw LJ, Petersen JW, Brown GH, Anderson RD, Shuster JJ, Cook-Wiens G, Rogatko A, Pepine CJ. A randomized, placebo-controlled trial of late $\mathrm{Na}$ current inhibition (ranolazine) in coronary microvascular dysfunction (CMD): Impact on angina and myocardial perfusion reserve. Eur Heart J 2016;37(19):15041513.

46. Russo G, Di Franco A, Lamendola P, Tarzia P, Nerla R, Stazi A, Villano A, Sestito A, Lanza GA, Crea F. Lack of effect of nitrates on exercise stress test results in patients with microvascular angina. Cardiovase Drugs Ther 2013;27(3):229-234.
47. Titterington JS, Hung OY, Wenger NK. Microvascular angina: An update on diaǵnosis and treatment. Future Cardiol 2015;11(2):229-242.

48. Agrawal S, Mehta PK, Bairey Merz CN. Cardiac syndrome X : Update. Heart Fail Clin 2016;12(1):141-156. http://dx.doi. org $/ 10.1016 / j . h f c .2015 .08 .012$. 